\title{
Hormonal Therapy in the Management of Premenstrual Syndrome
}

\author{
Jeffrey D. Tiemstra, MD, and Krishna Patel, PharmD
}

Background: Premenstrual syndrome (PMS) is characterized by any of a number of physical and psychological symptoms consistently occurring in the late luteal phase. Progesterone therapy is often recommended based on anecdotal evidence, although controlled studies have shown it to be ineffective. Oral contraceptives are more often used with mixed results. When hormonal therapy for PMS is indicated, the most appropriate choice remains a challenge.

Methods: We describe a case report of successful hormonal therapy for PMS and a review of the literature on the effectiveness of hormonal therapies.

Results and Conclusions: Estrogen is clearly effective in relieving symptoms of PMS, whereas progesterone is ineffective and might even worsen symptoms. Combination oral contraceptives are effective, undoubtedly as a result of the estrogen component. While little comparative data exist to guide choice of an oral contraceptive, maximizing the relative estrogenic potency of the oral contraceptive seems logical. Depressive symptoms might not respond to hormonal treatment, and new research suggests that selective serotonin reuptake inhibitors might be particularly effective. (J Am Board Fam Pract 1998;11:378-81.)

Premenstrual syndrome (PMS), also referred to as late luteal phase dysphoric disorder, is a problem commonly encountered in primary care, with up to 10 percent of women of childbearing age experiencing symptoms severe enough to seek medical treatment. ${ }^{1}$ Despite decades of research, standard criteria for the diagnosis of PMS are still debated. PMS can include any number of a wide variety of symptoms, such as depression, mood lability, swelling, abdominal pain, breast tenderness, headaches, or fatigue. Although the constellation of symptoms will vary from patient to patient, characteristic of PMS is the timing of symptoms around the menstrual cycle: symptoms predictably occur during the luteal phase, subside with menses, and are absent during the follicular phase.

Management of this common syndrome remains a challenge because many popular treatments are unproved or known to be ineffective. Noripharmacologic recommendations often include high-carbohydrate diets, vitamin and mineral supplementation (particularly pyridoxine),

Submitted, revised, 24 Sept 1997.

From the Family Practice Residency, Saint Joseph Medical Center (JDT), Joliet, and the Department of Pharmacy Practice, University of Illinois at Chicago (KP), Ill. Address reprint requests to Jeffrey D. Tiemstra, MD, Family Practice Residency, 24024 W. Brancaster Dr, Naperville, IL 60564. elimination of caffeine, biofeedback, and relaxation therapy. Because the beneficial effects of these measures have not been proved, patients who seek medical attention for this syndrome will usually require pharmacologic treatments. Patients with few or closely related symptoms might benefit from medication targeted at those symptoms. For instance, headaches, cramping, and abdominal pain can benefit from nonsteroidal anti-inflammatory drugs; abdominal bloating and leg edema will respond to loop diuretics; severe emotional symptoms will respond to antidepressants or anxiolytics; and migraine-type headaches can respond to migraine prophylactic or abortive therapy. Nevertheless, many patients either have too many symptoms to be targeted individually or have systemic symptoms such as fatigue for which there is no specific therapy. ${ }^{2-4}$

As a result of the temporal relation of PMS to the menstrual cycle, much attention has been given to estrogen and progesterone treatment, with the expectation that the entire array of PMS symptoms might respond. Results have been mixed, however, and misconceptions persist among both patients and providers regarding the relative efficacies of estrogen and progesterone therapy. Specifically, progesterone continues to be widely used in treatment despite convincing evidence that it is not 
beneficial, whereas estrogen remains underrecognized as effective therapy. The following case illustrates some of these pitfalls.

\section{Illustrative Case}

A 38-year-old woman came to the office for management of PMS symptoms. The patient described fatigue, sore throat, cold sores, myalgias, and chills starting in the week before her menstrual period and peaking in the first 2 days of her period. By days 3 and 4 her symptoms completely resolved, and she would feel fine for the next 2 to 3 weeks. She specifically denied headaches, bloating, swelling, or weight gain. Her menstrual flow was heavy (eight or more heavy pads) the first 3 days, tapering within 3 more days. These symptoms had developed within the last 5 years; before that she had more severe cramping with her periods but less severe systemic symptoms. During the past 3 years she had sought medical care repeatedly for this condition. Dietary modifications included low-fat, high-complex-carbohydrate diet, elimination of caffeine, and supplementation with a Bcomplex vitamin. Pharmacologic treatments had included ibuprofen, naproxen, cyclic progesterone, and most recently an oral contraceptive (Lo/Ovral) for 3 months. None of these treatments had produced any relief.

Recent evaluations had included physical examination, pelvic sonogram, complete blood count, iron studies, monospot test, thyroid function tests, and glucose tolerance tests, the results of which were normal.

The patient's prescription for oral contraceptives was changed from a $0.3-\mathrm{mg}$ norgestrel/0.03estradiol combination (Lo/Ovral) to a $1.0-\mathrm{mg}$ norethindrone/0.05-mg mestranol combination (Ortho-Novum 1/50) to achieve greater estrogenic activity relative to progestational activity. With this change the patient had complete resolution of her symptoms within 2 months and was very pleased with the result.

\section{Causes of PMS}

Because PMS symptoms are by definition temporally related to the menstrual cycle, it seems logical that cyclic fluctuations in estrogen or progesterone would be causally related. Studies in the 1980 s, however, found that estrogen and progesterone levels of patients with PMS did not differ from asymptomatic control patients, and that in individ- ual patients neither the timing nor the severity of symptoms correlated with fluctuations in hormone levels. ${ }^{5,6} \mathrm{~A} 1991$ study confirmed that variations in hormone levels did not correlate with symptoms, and additionally found that pharmacologically terminating the luteal phase did not prevent the normal cyclic appearance of PMS symptoms. ${ }^{7}$ Thus although PMS symptoms correlate with the late luteal phase of the menstrual cycle, luteal phase levels of estradiol and progesterone do not appear to be direct causative factors.

More recent hypotheses on the cause of PMS suggest a role for fluctuating levels of neurotransmitters in the production of symptoms. ${ }^{8,9}$ Specifically, estrogen has been found to stimulate certain populations of dopamine and serotonin receptors, the same receptors that are stimulated by selective serotonin reuptake inhibitor (SSRI) antidepressants. ${ }^{10}$ In addition, antiestrogen drugs, such as tamoxifen and progesterone, have been found to stimulate PMS symptoms in susceptible patients. ${ }^{11}$ Thus, PMS symptoms appear to be mediated in the central nervous system, and declining levels of estrogen in the late luteal phase might indirectly contribute to the timing of their appearance.

Because of the decline of both estrogen and progesterone levels in the late luteal phase coincident with the timing of PMS symptoms, both have been considered and studied as potential treatments. New understanding of the role of neurotransmitters in PMS has helped explain what have been in the past confusing and conflicting data.

\section{Treatment}

\section{Progesterone}

Anecdotal case reports suggested that progesterone might be of benefit in the treatment of PMS. Controlled studies, however, have consistently shown no benefit from progesterone; in fact, several studies have found a worsening of symptoms with progesterone. ${ }^{12-14}$ Unfortunately, it continues to be recommended as an option worth trying. Case reports of responses to progesterone treatment are most likely explained by the fairly high placebo response seen in many PMS studies (up to 50 percent), ${ }^{9}$ although weak estrogenic effects of therapeutic doses of progesterone through peripheral conversion to estrogen are theoretically possible.

With the increasing use of long-acting forms of progesterone for contraception (Depo-Provera, Norplant), physicians should be aware of the po- 
tential for these treatments to aggravate preexisting PMS. Women with PMS symptoms of mood changes, food cravings, sleep disturbances, and dysmenorrhea might experience worsening of symptoms with these progesterone-only forms of contraception.

\section{Estrogen}

Given the new information on the effect of estrogen on neurotransmitters, it is not surprising that estrogen is effective in the treatment of PMS. Continuous estrogen therapy has consistently proved effective in relieving PMS symptoms, whether orally posthysterectomy, ${ }^{15}$ transdermally, ${ }^{16}$ or through implants. ${ }^{17}$ Unfortunately, the addition of cyclic progesterone to these regimens results in a reduction of the beneficial effect. Because the carcinogenic potential of unopposed estrogen precludes its use for most women, the usefulness of estrogen as a treatment of PMS is compromised by the need to add cyclic progesterone.

\section{Oral Contraceptives}

Combination oral contraceptives have both estrogenic and progestational effects. Whereas the relative strengths of these effects can be approximated for individual pill combinations by the incidence of various side effects, the relative effects of any given combination on an individual patient are less predictable. Relative absorption of the estrogen and progesterone, peripheral conversion of both hormones, and individual susceptibility to specific side effects can all determine the patient's response. Because the beneficial effect of oral contraceptives on PMS is most likely due to the estrogen effect, it is not surprising that combination oral contraceptives have produced less dramatic results in the treatment of PMS than estrogen alone.

Both observational ${ }^{18-21}$ and controlled prospective $^{22-24}$ studies have generally found that oral contraceptive users have fewer PMS symptoms than nonusers. Symptoms likely to respond include more specific physical symptoms such as bloating, headaches, abdominal pain, and breast tenderness. Less clear has been the effect of oral contraceptives on such emotional symptoms as irritability and depression; some studies have suggested that PMSrelated depression might not respond as well as physical symptoms to oral contraceptives. ${ }^{20,22}$

Although it is reasonable to conclude that oral contraceptives with relatively greater estrogenic effect will be more beneficial for PMS patients, there are few data to guide the physician's choice of a particular oral contraceptive. Studies looking at monophasic and triphasic formulations individually have found positive effects for both. One study that compared monophasic with triphasic formulations included eight different pill formulations in their two groups; no significant differences were found in symptoms, with the exception of breast tenderness (improved more in monophasic group). ${ }^{24}$ To date no controlled studies have been done comparing a specific combination in different ratios to assess the effect of varying the estrogen-progesterone ratio.

Given the current data on hormone therapy for PMS, four conclusions can reasonably be drawn regarding choosing an oral contraceptive: (1) the initial choice should be based on suitability to the individual patient rather than that PMS is being treated; (2) given the variability in response rates, if the initial choice is ineffective, it is reasonable to try different combinations before abandoning hormonal therapy; (3) when there is no clear-cut first choice, or when a change in oral contraceptive choice is indicated, a combination with a relatively higher estrogen activity and minimal progesterone activity might be more effective in treating PMS symptoms, with the possible exception of breast tenderness; and (4) PMS-related depressive symptoms might not respond well to hormonal therapy. If depressive symptoms are the only symptoms unresponsive to an oral contraceptive, adding an SSRI-type antidepressant might be preferable to switching the oral contraceptive.

\section{Conclusion}

Premenstrual syndrome is a common patient problem in primary care. Many patients will come to their family physician for treatment, often when nonpharmacologic treatment has failed. Symptom-specific therapy can provide relief for some patients, but hormone therapy should be considered when a wide variety of symptoms are described, when symptom-specific therapy is inadequate, or when contraception is desired.

Progesterone therapy should be avoided, because progesterone is no better than placebo and can actually worsen the condition. Unopposed estrogen therapy can be considered in patients who are not at risk for endometrial cancer. For most women, however, combination oral contraceptives 
will be the hormonal therapy of choice. As initial therapy any monophasic or triphasic low-dose oral contraceptive is appropriate. If a response is not seen initially, different combinations with increasing relative estrogenic activity can be tried before abandoning oral contraceptive therapy altogether. When prescribing oral contraceptives, physicians should keep in mind the common risks and complications, particularly the increased risk of cardiovascular complications for women 30 to 50 years of age.

If the response to oral contraceptives is inadequate, other hormonally directed therapies such as danazol, gonadotropin-releasing hormone analogues, and oophorectomy have clearly proved effectiveness, but their side effects and long-term consequences are greater, and they should generally be reserved for only the most extreme cases. ${ }^{1}$

One recent study found that fluoxetine was effective in the treatment of premenstrual dysphoria. ${ }^{25}$ This finding, combined with a better understanding of the cause of PMS, suggests that for patients with predominantly emotional symptoms, an SSRI antidepressant might be a better initial treatment choice than hormonal therapy.

\section{References}

1. Hsia LS, Long MH. Premenstrual syndrome. Current concepts in diagnosis and management. J Nurse Midwifery 1990;35:351-7.

2. Severino SK, Moline ML. Premenstrual syndrome. Identification and management. Drugs 1995;49:7182.

3. DeMonico SO, Brown CS, Ling FW. Premenstrual syndrome. Curr Opin Obstet Gynecol 1994;6:499. 502.

4. Parker PD. Premenstrual syndrome. Am Fam Physician 1994;50:1309-17.

5. Rubinow DR, Hoban MC, Grover GN, Galloway DS, Roy-Bryne P, Andersen R, et al. Changes in plasma hormones across the menstrual cycle in patients with menstrually related mood disorders and in control subjects. Am J Obstet Gynecol 1988;1 58:5-11.

6. Backstrom T, Sanders D, Leask R, Davidson D, Warner P, Bancroft J. Mood, sexuality, hormones, and the menstrual cycle. II. Hormone levels and their relationship to the premenstrual syndrome. Psychosom Med 1983;45:503-7.

7 Schmidt PJ, Neiman LK, Grover GN, Muller KL, Merriam GR, Rubinow DR. Lack of effect of induced menses on symptoms in women with premenstrual syndrome. N Engl J Med 1991;324:1174-9.

8. Smith S, Schiff I, editors. Modern management of premenstrual syndrome. New York: WW Norton \& Company, 1993.

9. Mortola JF. Issues in the diagnosis and research of premenstrual syndrome. Clin Obstet Gynecol 1992; 35:587-98.

10. Fink G, Sumner BE, Rosie R, Grace O, Quinn JP. Estrogen control of central neurotransmission: effect on mood, mental state, and memory. Cell Mol Neurobiol 1996;16:325-44.

11. Arpels JC. The female brain hypoestrogenic continuum from the premenstrual syndrome to menopause. A hypothesis and review of supporting data. J Reprod Med 1996;41:633-9.

12. Moline ML. Pharmacologic strategies for managing premenstrual syndrome. Clin Pharm 1993;12:181-96.

13. Freeman E, Rickels K, Sondheimer SJ, Polansky M. Ineffectiveness of progesterone suppository treatment for premenstrual syndrome. JAMA 1990;264:349-53.

14. Sampson GA. Premenstrual syndrome: a doubleblind controlled trial of progesterone and placebo. $\mathrm{Br}$ J Psychiatry 1979;135:209-15.

15. Casper RF, Hearn MT. The effect of hysterectomy and bilateral oophorectomy in women with severe premenstrual syndrome. Am J Obstet Gynecol 1990; 162:105-9.

16. Watson NR, Studd JW, Savvas M, Garnett T, Baber RJ. Treatment of severe premenstrual syndrome with oestradiol patches and cyclical oral norethisterone. Lancet 1989;2:730-2.

17. Magos AL, Brincat $M$, Studd JW. Treatment of the premenstrual syndrome by subcutaneous estradiol implants and cyclical oral norethisterone: placebocontrolled study. Br Med J Clin Res Ed 1986;292: 1629-33.

18. Bancroft J, Rennie D. The impact of oral contraceptives on the experience of perimenstrual mood, clumsiness, food craving and other symptoms. J Psychosom Res 1993;37:195-202.

19. Brown $S$, Vessey $M$, Stratton I. The influence of method of contraception and cigarette smoking on menstrual patterns. Br J Obstet Gynaecol 1988;95: 905-10.

20. Kutner SJ, Brown WL. Types of oral contraceptives, depression, and premenstrual symptoms. J Nerv Ment Dis 1972;155:153-62.

21. Herzberg B, Coppen A. Changes in psychological symptoms in women taking oral contraceptives. $\mathrm{Br}$ J Psychiatry 1970;116:161-3.

22. Graham CA, Sherwin BB. A prospective treatment study of premenstrual symptoms using a triphasic oral contraceptive. J Psychosom Res 1992;36:257-66.

23. Backstrom $T$, Hansson-Malmstrom $Y$, Lindhe $B A$, Cavalli-Bjorkman B, Nordenstrom S. Oral contraceptives in premenstrual syndrome: a randomized comparison of triphasic and monophasic preparations. Contraception 1992;46:253-68.

24. Walker A, Bancroft J. Relationship between premenstrual symptoms and oral contraceptive use: 2 controlled study. Psychosom Med 1990;52:86-96.

25. Steiner M, Steinberg S, Stewart D, Carter D, Berger C, Reid R, et al. Fluoxetine in the treatment of premenstrual dysphoria. Canadian Fluoxetine/Premenstrual Dysphoria Collaborative Study Group. N Engl J Med 1995;332:1529-34. 